\title{
The Effects of Colchicine and Auxin on Rhizoid Formation of Dryopteris erythrosora
}

\author{
by Yukio KATO* \\ 加藤幸雄*： ベニシダの仮根形成に対するコルヒチン及びオーキシンの影響
}

Received April 13, 1957

It is a well known fact that auxin and colchicine affect both the growth of elongation and its morphological type. The author stated in a separate report of this series that in Equisetum indole acetic acid increases the number of rhizoids in the germinating spore, while in Osmunda it acts as a kind of inhibitor for the rhizoid formation (Kato, in press). The present study was undertaken to determine the effects of growth hormone (naphthalene acetic acid) and colchicine on rhizoid formation in Dryopteris spores. Regarding the influence of colchicine on young prothallium, Yamazaki (7) reported on Polystichum craspedorum, but not on the role of colchicine for the rhizoid-differentiation.

The spores used in the present experiment were taken from the leaves of Dryopteris erythrosora grown in the vicinity of Nagoya City. Spores were cultured for 15-20 days in one of the following concentrations of $\alpha$-naphthalene acetic acid (NAA); $0.01,0.1,1,10,100$ p.p.m. Aqueous solutions of colchicine were prepared in concentrations from 0.2 to $0.05 \%$. As control, 1/5 dilute Knop's agar medium was applied. The method used in the present experiment was the same as that described in the separate report.

\section{Results}

In the control medium, after seven days of culture, the first division of spores takes place and the rhizoids grow out of the exine membrane (Fig. 1g.)

1. The effects of NAA

In the NAA-containing medium (100 p.p.m.), the first division of spores is strongly inhibited and spores are greatly enlarged prior to the cell division. That is to say, spores grow not only longitudinally but also laterally. Cwing to this hypertrophy of spores, the exine membrane is frequently cast off (Fig. 1a). The spore elongates longitudinally and becomes oblong in shape.

In the control medium, the germinating spore becomes 9 or 10 cells in the 20 day-old material and the growth is usually in two dimensions. The spore cultured in the medium containing NAA often consists of a single elongated cell. No cell

* Biological Institute, Faculty of Science, Nagoya University, Nagoya. 名古屋大学理学部生物学教空 
division occurs. Figure 1b shows the spore consisting of two cells without rhizoiddifferentiation. The division seems to occur in a position perpendicular to the axis of polarity. Figure 1c shows also a spore without rhizoid. The new cell wall of the first division seems to be at right angles to the axis of polarity. Even if the rhizoids
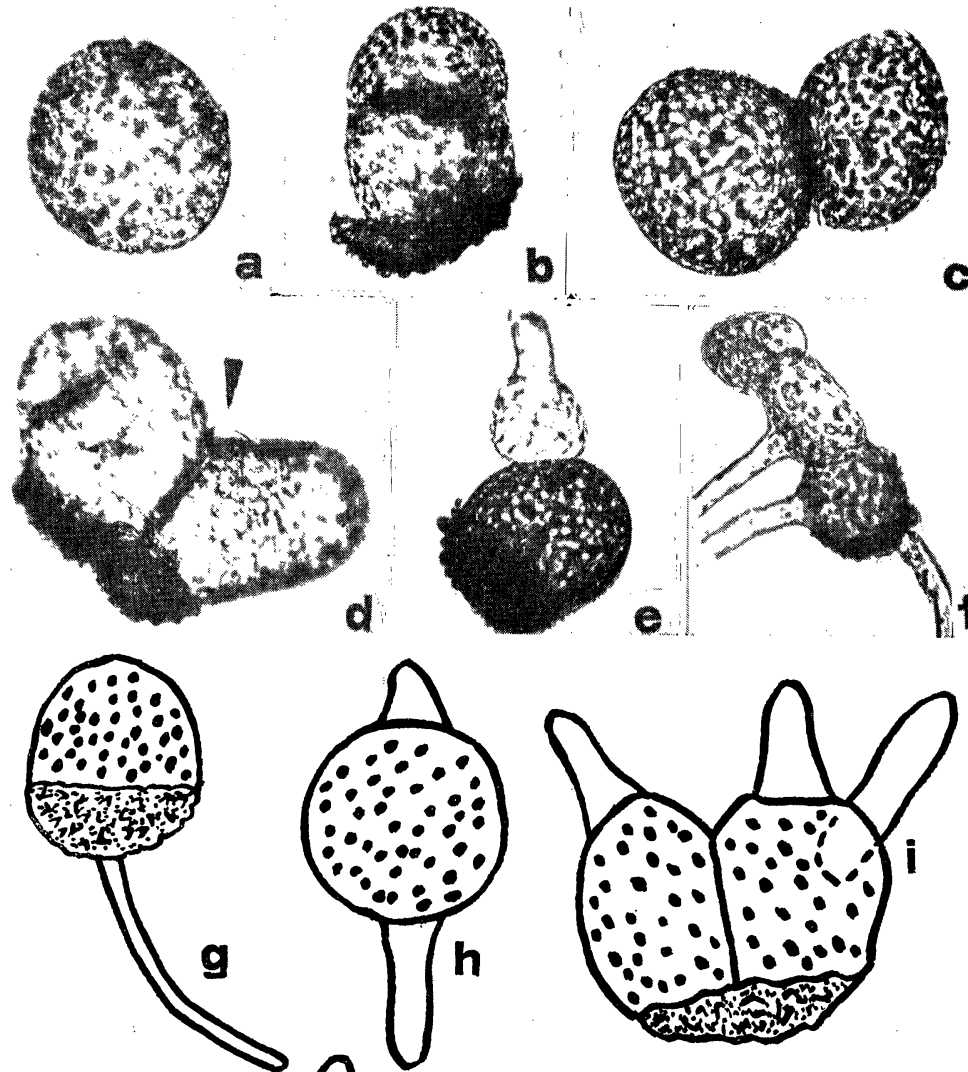

d $e$
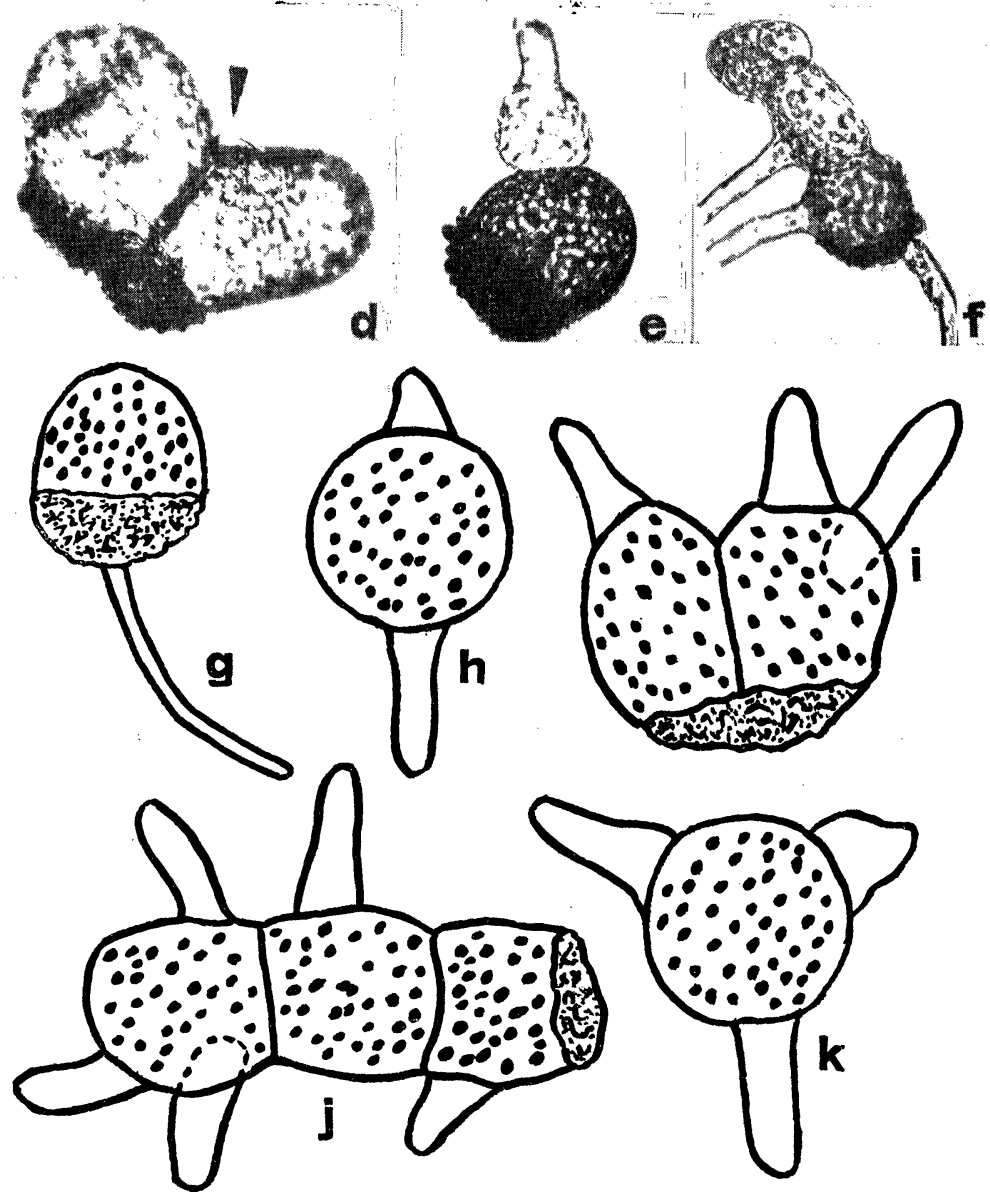

\section{.}




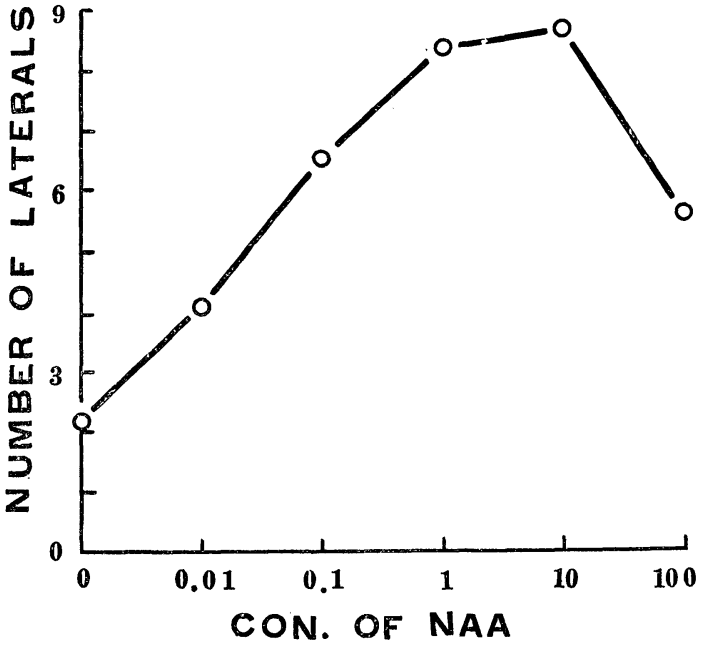

Fig. 2. Relation between concentrations of NAA and average number of lateral rhizoids per protonema (15-day-old material).

do differentiate, their elongation is extremely inhibited. Sometimes, the rhizoid is observed as a small protuberance (Fig. 1d). The swelling of the rhizoid is one of the effects of auxin (Fig. 1h-k). The rhizoid can originate not only from the rhizoidal pole but also from the protonematic pole (Fig. 1d and e). This may be regarded as a kind of lateral rhizoids rather than a reversal of the polarity. Lateral rhizoids are not usually formed up to the 5 or 6 cell stage of protonema in the control medium, while 1-5 laterals are formed even in the 1 or 2 cell stage in the NAA-containing medium.

The relation between

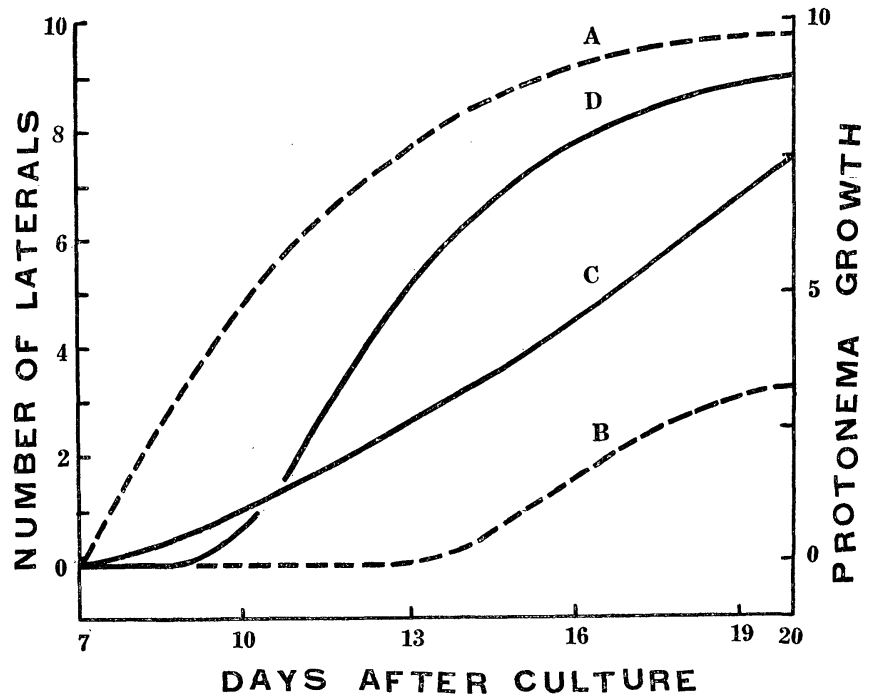

Fig. 3. Relation between protonema growth and average number of lateral rhizoids per protonema.

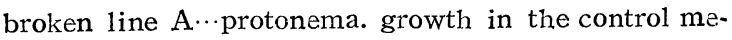
dium. broken line $\mathrm{B} \cdots$ average number of laterals. unbroken line $\mathrm{C} \cdots$ protonema growth in the medium containing NAA (10 p.p.m.). unbroken line D... average number of laterals in the medium contain. ing NAA. the used concentrations of NAA and the average num. ber of lateral rhizoids is shown in Figure 2. This result was obtained from the 15-day-old material. The number of laterals is greatly increased in concentrations of $0.1-10 \mathrm{p} . \mathrm{p}$. m. of NAA. In Figure 3 the protonema growth and the average number of laterals in the control medium and in the medium containing NAA are shown. From the figure presented here, it may be concluded that in the control medium there is a parallel relation between protonema growth and the number of laterals, i. e., the more the proto- 
nema grows or the cell number increases the more the average number of laterals per protonema increases. First laterals appear, for instance, on about the 15th day of culture, when the protonema grows to $8.7 \mathrm{~mm}$. On the other hand, laterals emerge on the 9 th day of culture, when the protonema grows to $0.5 \mathrm{~mm}$ in the medium containing NAA. As mentioned above, in an extreme case, a lateral rhizoid is formed even in one cell stage of an undivided spore (Fig. $1 \mathrm{~h}$ and $1 \mathrm{k}$ ). It is, therefore, evident that NAA acts as an effective inducer for the formation of lateral rhizoid. In conclusion, NAA treatment of the germinating spores prevents the formation of rhizoid from the spore and promotes the formation of lateral rhizoid from the protonema. It is possible to assume that the formation of lateral rhizoid from the protonema is different from that of rhizoid from the spore in respect to the morphogenetic factor. NAA induces not only the swelling of rhizoid, but also of protonematic cells. Thereore, the growth and arrangement of protonematic cells are very irregular. The division axis of protonematic cells also varies in its pattern.
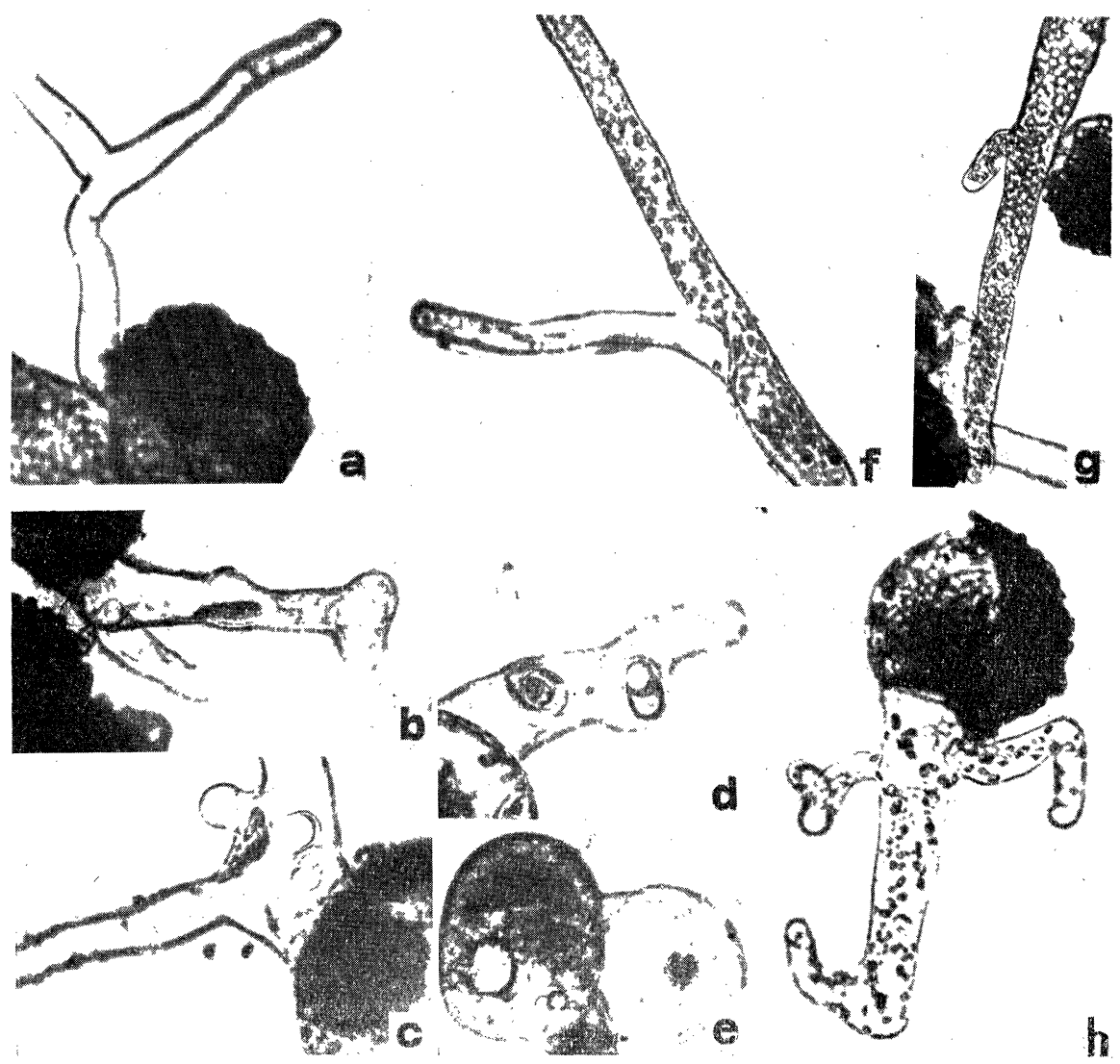

Fig. 4a-h. Abnormally branching rhizoids and rhizoidal protonemata induced by colchicine. a-d, ramification of rhizoids. e, swelling of rhizoid. $\mathrm{f}$, rhizoid formation from the rhizoidal protonema. $g$, ramification of rhizoidal protonema. $h$, ramified rhizoidal protonema and protonema appearing from the exine membrane. 
II. The effects of colchicine

The author (4) described previously that the Equisetum spore cultured on a medium containing a large quantity of colchicine develops into a giant globe of about 10 to 15 times its normal volume, cell division being perfectly inhibited, and that cell-differentiation does not eventually occur. It is thus clear that colchicine acts not only as a polyploid inducer, but also as an effective modifier of rhizoiddifferentiation and morphogenesis. In the case of Dryopteris spores, the rhizoids are always capable of differentiating, even in higher concentrations of colchcine. The rhizoidal protonema occurs in a higher frequency. The rhizoidal protonema is more lanky and longer containing less chloroplasts than normal protonema (5). This phenomenon was also observed in the medium containing tryptophane. Ramification of rhizoids is another effect of colchicine. Some photomicrographs (Fig. 4) show the germinating spores affected by colchicine. Figure $4 \mathrm{a}-\mathrm{d}$ represent the typical ramification of rhizoids, $4 \mathrm{e}$ swelling rhizoid, $4 \mathrm{f}$ rhizoid formation of the rhizoidal protonema, $4 \mathrm{~g}$ ramified rhizoidal protonema, and $4 \mathrm{~h}$ the spore with a protonematic cell which emerges from the exine membrane and is exhibiting a characteristic state of ramified rhizoidal protonema. More detailed studies on the ramification of rhizoid induced by colchicine were reported previously (6).

\section{Discussion}

According to Bloch $(1,2)$, cell-differentiation is determined by two major ways; one of these is the ability of specific internal and external environmental conditions eliciting a particular path of differentiation from previously undifferentiated, totipotent cells. The other type of differentiation is determined at a given cell division and a new characteristic then persists through subsequent cell division without apparent dependence on a specific environment. An example of the latter type is found in the case of rhizoid formation in ferns. The differentiation of cells in the meristem of Ricinus into secretory idioblasts has been described by Bloch (2). The first division causes an undifferentiated cell to divide into two unlike daughters, the one containing a red pigment, tannin and unsaturated fats, and other lacking detectable amounts of these substances. The pigmented daughter cell produces also a row of pigmented cell; the colourless daughter cell gives rise to a row of cells, all of which are also pigmented. In ferns the daughter cell originating at the pole of the mother cell rich in protoplasm is characterized by its larger size and an increased power of differentiation, i.e., a capability of more active nuclear division.

Jacobs (3) reported that auxin controls the rhizoid formation in Bryopsis. The author obtained similar results in the germinating spore of Equisetum treated by IAA. In Dryopteris, IAA induces without exception the rhizoid to develop, causing it to swell. NAA inhibits frequently the rhizoid formation form the spore, but promotes the formation of lateral rhizoids. On the other hand, in Osmunda NAA prevents the rhizoid formation from both the spore and protonema. Therefore, the 
action of auxin varies greatly according to the spore's condition in the different species and even in one and the same species used as an experimental material. General aspects of colchicine effect of fern prothallia are not touched in the present paper.

\section{Summary}

The effects of naphthalene acetic acid and colchicine on the rhizoid formation in Dryopteris spore were described. The results obtained are summarized as follows:

1. In contrast with the case of Equisetum spore, the formation of rhizoid is prevented greaty in the medium containing NAA. That is, NAA-treatment of the germinating spores prevents the formation of rhizoid from the spore, but rather promotes it from the protonema.

2. The position of the rhizoid initiation is very irregular in the spore cultured in the medium containing NAA.

3. The frequency of occurrence of the rhizoidal protonema is higher in the case of the colchicine treatment.

The author wishes to express his hearty thanks to Prof. T. Shimamura who kindly examined the manuscript of the present paper.

\section{References}

1) Bloch, R., Science, $106: 321-322$ (1947). 2) — Growth 12:271-284 (1948). 3) Jacobs, Wm. P., Biol. Bull. 101 : 300-305 (1951). 4). Kato, Y., Biol. Sci. 7: 153-156 (1955). 5) -_, Фyton (in press). 6) - Bot. Mag. Tokyo 70: 209-216 (1957). 7) Yamazaki, N., Cytologia 19 : 249-245 (1954). 